\title{
On the Morse-Smale characteristic of a differentiable manifold
}

\section{George M. Rassias}

In this note, the Morse-Smale characteristic of a differentiable manifold is defined and certain of its properties are studied.

Let $M$ be a closed (that is, compact without boundary) $C^{\infty}$ differentiable manifold and $f: M \rightarrow R$ be a $C^{\infty}$ differentiable function on $M$. A point $p \in M$ is a critical point of $f$, if and only if, the induced map

$$
f_{*}: T M_{p} \rightarrow T R_{f(p)}
$$

is zero, where $T M_{p}$ is the tangent space of $M$ at $p$. A real number $a \in R$ is a critical value of $f$ if $f^{-1}(a)$ contains a critical point of $f$. If $f^{-1}(a)$ contains no critical points, then $a$ is a regular value of $f$. A critical point $p$ of $f$ is said to be non-degenerate if and only if the matrix

$$
\left.\int\left(\partial^{2} f / \partial x_{i} \partial x_{j}\right)(p)\right)
$$

is non-singular. This matrix defines a symetric bilinear form on the tangent space $T M_{p}$. This bilinear form is the Hession of $f$ at $p$. The index of a critical point $p$ of $f$ is the maximal dimension of a subspace of $T M_{p}$ on which the Hessian of $f$ is negative definite.

A $C^{\infty}$ differentiable function $f: M+R$ is said to be a Morse function if $f$ has only non-degenerate critical points.

Received 15 March 1979. 
DEFINITION. Let $M$ be a closed $C^{\infty}$ differentiable manifold of dimension $n$. The Morse-Smale characteristic of $M$, denoted by $\mu(M)$, is

$$
\mu(M)=\min _{f \in \Omega} \sum_{i=0}^{n} c_{i}(M, f),
$$

where $\Omega$ is the space of Morse functions on $M$, and $c_{i}(M, f)$ is the number of critical points of index $i$ of $f$ in $\Omega$.

THEOREM. Let $M$ be a closed $C^{\infty}$ differentiable manifold, $\operatorname{dim} M=n<4$. Then

$$
\mu(M)=\min _{f \in \Omega} \sum_{i=0}^{n} c_{i}(M, f)=\sum_{i=0}^{n} \min c_{i \in \Omega}(M, f) .
$$

Proof. If $n=1$ it is obviously true. If $n=2$, it can be also proved that the equality holds.

If $n=3$, then by Smale [1], [2], there exists a Morse function $f$ on $M^{3}$ having a single critical point of index 0 , and a single one of index 3 ; that is,

$$
c_{0}\left(M^{3}, f\right)=c_{3}\left(M^{3}, f\right)=1
$$

However, the Euler characteristic of $M^{3}$ equals zero. Thus $c_{1}\left(M^{3}, f\right)=c_{2}\left(M^{3}, f\right)$ because of the last (equality) of the Morse inequalities. Hence

$$
\begin{aligned}
\min _{f \in \Omega} \sum_{i=0}^{3} c_{i}\left(M^{3}, f\right)=\min _{f \in \Omega}\left(2+2 c_{1}\left(M^{3}, f\right)\right)=2+2 \min _{f \in \Omega} c_{1}\left(M^{3}, f\right) & \\
& =\sum_{i=0}^{3} \min _{f \in \Omega} c_{i}\left(M^{3}, f\right) .
\end{aligned}
$$

PROBLEM. Find necessary and sufficient conditions on $M^{n}, n \geq 4$, so that

$$
\mu\left(M^{n}\right)=\min _{f \in \Omega} \sum_{i=0}^{n} c_{i}\left(M^{n}, f\right)=\sum_{i=0}^{n} \min _{f \in \Omega} c_{i}\left(M^{n}, f\right)
$$

Using an argument similar to that of the previous theorem, in particular 
the fact that the Euler characteristic of any closed odd-dimensional manifold equals zero, the following proposition can be proved.

PROPOSITION. The Morse-Smale characteristic of any closed $c^{\infty}$ differentiable odd-dimensional monifold is an even integer greater than or equal to 2 .

REMARK. It is not known yet for which closed $C^{\infty}$ differentiable manifolds $M, N$,

$$
\mu(M \times N)=\mu(M) \cdot \mu(N) .
$$

(of course, $\mu(M \times N) \leq \mu(M) \cdot \mu(N)$.) If this is the case, then $\mu\left(H^{3}\right)=2$ where $H^{3}$ is any homotopy 3-sphere and so the Poincaré conjecture would be true since $\mu\left(H^{3} \times H^{3}\right)=4$. In particular, the Poincare conjecture is true, if and only if, $\mu\left(H^{3}\right) \leq \mu\left(H^{3} \times H^{3}\right)$.

\section{References}

[1] Stephen Smale, "Generalized Poincaré's conjecture in dimensions greater than four", Am. of Math. (2) 74 (1961), 391-406.

[2] S. Smale, "On the structure of manifolds", Amer. J. Math. 84 (1962), $387-399$.

Ф.E.A.A.,

279 Patision Street,

Athens,

Greece. 